\title{
Formation of prismatic loops from C15 Laves phase interstitial clusters in body-centered-cubic iron
}

\author{
Yongfeng Zhang1, Xian-Ming Bai, Michael R. Tonks, S. Bulent Biner \\ Fuels Modeling and Simulation, Idaho National Laboratory (INL), Idaho Falls, ID 83415, USA
}

\begin{abstract}
This work reports the transformation of C15 Laves phase interstitial clusters to prismatic loops in bodycentered-cubic iron. Molecular dynamics simulations and elasticity analysis show that within a certain size range, $\mathrm{C} 15$ clusters are more stable than loops, but the relative stabilities are reversed beyond this range. Consistently, C15 clusters can grow by absorbing interstitials at small sizes and transform into loops later. Both $\langle 100\rangle$ and $\langle 111\rangle / 2$ loops may result from the transformation, revealing a new formation mechanism for $\langle 100\rangle$ loops.
\end{abstract}

Radiation by high-energy particles produces vacancies and self-interstitial-atoms (SIAs) in materials, and their agglomerates including voids and prismatic loops, leading to swelling and hardening. In bcc Fe, SIA clusters at finite sizes usually form loops with either a $\langle 100\rangle$ or a $\langle 111\rangle / 2$ (referred to as $\langle 111\rangle)$ Burgers vector $[1,2]$, with the former being more prevalent at high temperatures as shown by previous theoretical analysis [3] and experiments [4]. $\langle 111\rangle$ loops are highly mobile along their Burgers vector directions so that they may quickly move to nearby sinks [5-7]. In contrast, $\langle 100\rangle$ loops have much higher migration barriers [8], and can cause detrimental effects as stationary sinks for point defects. While there is abundant evidence of $\langle 100\rangle$ loop formation, how they actually form remains a puzzle. Due to the high formation energy of $\langle 100\rangle$ SIA dumbbell compared to the most stable $\langle 110\rangle$ dumbbell [9, 10], direct nucleation of $\langle 100\rangle$ loops by SIA clustering is highly unlikely. Instead, their formation has been explained by the interaction of two $\langle 111\rangle$ loops $[8,11]$ or direct transition from a single $\langle 111\rangle$ loop [12]. However, the proposed interaction mechanism requires the encounter of two $\langle 111\rangle$ loops with similar sizes and overlapping glide cylinders [8], make it unlikely in reality. Instead, absorptions of smaller $\langle 111\rangle$ loops by larger ones are usually observed experimentally when they do Zhang)

Email address: yongfeng.zhang@inl.gov (Yongfeng

Preprint submitted to Elsevier meet [13]. The detailed mechanism responsible for the transition from a single $\langle 111\rangle$ to a $\langle 100\rangle$ loop was unclear. The recently proposed crowdion reorientation mechanism [14] seems to be challenging at finite loop sizes, since a junction has to form when the loop is partially reoriented, leading to drastic increase in total loop length and energy.

Besides loops, three-dimensional (3D) SIA cluster have also been predicted in bcc Fe by molecular dynamics (MD) simulations $[15,16]$ and densityfunctional-theory (DFT) calculations [17]. More interestingly, some 3D SIA clusters may form the socalled C15 Laves phase, and are even more stable than loops at small sizes as shown by DFT calculations [18]. However, it is unclear if such stability persists at large sizes, although bulk $\mathrm{C} 15$ phase can be mechanically and dynamically stable [19]. Moreover, C15 clusters have never been reported experimentally, leaving their existence another puzzle.

In this work, the two aforementioned puzzles are addressed by MD simulations and are found to have a close correlation. C15 clusters are more stable than loops only within a certain size range. Consistently, dynamics simulations demonstrate that C15 clusters can grow by absorbing individual SIAs until they transform into loops at sizes below the resolution in previous experiments, explaining their absence there. Both $\langle 100\rangle$ and $\langle 111\rangle$ loops can result from this transformation, revealing a new mechanism for $\langle 100\rangle$ loop formation that does not require 


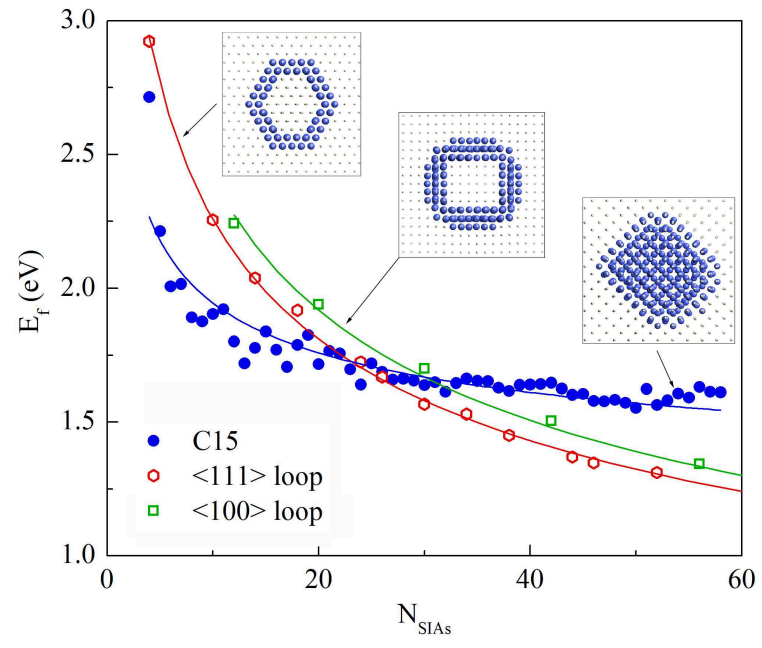

Figure 1: (Color online) Formation energies of C15 clusters and loops given by the M07 potential. The solid lines are fitted to Eqs. 1 and 2. The insets show the configurations for a $\langle 111\rangle$ loop, a $\langle 100\rangle$ loop and a C15 cluster. Bcc and non-bcc atoms identified by the CNA method are shown by grey dots and blue spheres respectively.

special constraints.

To investigate the relative stabilities between $\mathrm{C} 15$ clusters and loops, their formation energies are calculated with the M07 potential (See Appendix A and Table 10 in [10]. Note that the sign for parameter $B_{1}$ should be negative). This potential well reproduces DFT predictions on the energetics of small SIA clusters [10] and the energy landscape of C15 clusters relative to $\langle 111\rangle$ loops [18]. To create $\langle 111\rangle$ and $\langle 100\rangle$ loops, parallel $\langle 111\rangle$ and $\langle 100\rangle$ dumbbells are inserted on the $\{111\}$ and $\{100\}$ planes respectively. $\langle 111\rangle$ loops are hexagonal in shape, with the edges along $\langle 110\rangle$ directions. After relaxation, their residing plane changes from $\{111\}$ to $\{110\}$ plane. $\langle 100\rangle$ loops are square, with their edges along $\langle 110\rangle$ as well. C15 clusters are inserted as spherical inclusions with the C15 Laves structure, fully coherent with the bcc matrix. After construction, the systems are relaxed at $300 \mathrm{~K}$ for $50 \mathrm{ps}$ and then minimized to obtain the total energy $E_{t o t}$. The formation energy $E_{f}$, normalized by the number of SIAs $(N)$, is defined as $E_{f}=\left(E_{t o t}-E_{0}\right) / N$. Here, $E_{0}$ is the total energy of a perfect crystal with the same number of atoms. We use LAMMPS simulation package [20] for all simulations with the isothermal-isobaric (NPT) ensemble and periodic boundary conditions, and the common-neighboranalysis (CNA) [21] to identify defects from the bcc matrix.
As shown in Fig. 1, at small sizes (starting from 4 SIAs) C15 clusters are found to be energetically more favorable than loops, in agreement with DFT calculations [18]. However, beyond a critical size of about 20-30 SIAs, the relative stabilities are reversed. The crossover predicted by the M07 potential can also be predicted by elasticity theory. For a loop with an effective radius $R$, the formation energy $E_{f}$ (per SIA) can be estimated by: $[3,22]$

$$
E_{f}=2 \Omega\left(F_{t} \ln \frac{4 R}{e \delta}+F_{\delta}+F_{c}\right) / R b
$$

Here $\delta$ is the core width; $b$ is the Burgers vector; $\Omega$ is the atomic volume of bcc Fe; $e=2.71828 ; F_{t}, F_{\delta}$ and $F_{c}$ are the prelogarithmic factor for a straight dislocation, the anharmonic core and the core-traction energy.

For a C15 cluster, assuming a spherical, coherent inclusion in an isotropic matrix [23], $E_{f}$ is given by:

$$
E_{f}=6 \Omega \gamma / R+12 \mu \Omega \varepsilon^{2} / \alpha+3\left(E_{c o h}^{b c c}-E_{c o h}^{C 15}\right)
$$

Where $\gamma$ is the effective interfacial energy; $\mu$ is the shear modulus of the matrix; $\epsilon$ is the misfit strain; $\alpha$ is a constant given by the elastic moduli of bulk bcc and C15 phases; $E_{c o h}^{b c c}$ and $E_{c o h}^{C 15}$ are the corresponding cohesive energies. The three terms represent the interfacial energy, the elastic strain energy and the excess cohesive energy of C15 phase relative to bcc phase, respectively.

The formation energies of C15 clusters (Eq. 1) and loops (Eq. 2) exhibit distinct size dependences. For C15 clusters, the interfacial term decays with $1 / R$ and becomes negligible at large sizes. In contrast, the strain and excess cohesive energies, normalized by $N$, are size independent and their summation gives $E_{f}$ at infinite $R$, about $1.04 \mathrm{eV} / \mathrm{SIA}$ as determined by fitting the MD results to Eq. 2. The fitted result is close to that directly calculated ( 0.94 eV/SIA) using the parameters given by M07, showing the validity of Eq. 2. From the fitting $\gamma$ is found to be about $0.79 \mathrm{~J} / \mathrm{m}^{2}$. As for loops, $E_{f}$ decays approximately with $1 / R\left(\ln \left(\frac{4 R}{e \delta}\right) / R\right.$ for the first term), and it approaches 0 at infinite $R$. Therefore, loops are more stable than $\mathrm{C} 15$ clusters at large sizes, and a crossover occurs since C15 clusters are more stable at small sizes [18].

The formation energy of $\mathrm{C} 15$ clusters is much lower than that for a single SIA $(\sim 3.73 \mathrm{eV}[10])$, suggesting a high binding energy between them. Therefore, there is a strong tendency for C15 clusters to grow, yet they have not been observed experimentally. To investigate this, a number of growth 

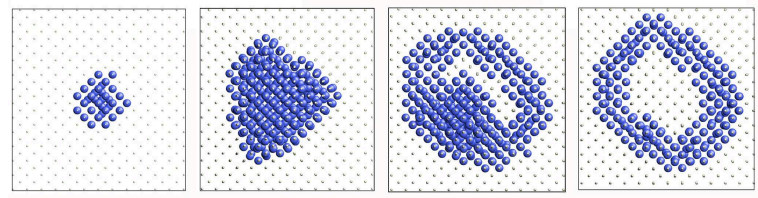

Figure 2: (Color online) Atomic configurations (projected along $\langle 100\rangle)$ showing the growth of a C15 cluster and subsequent transformation into a $\langle 100\rangle$ loop during a growth simulation. from left to right, the system contains $6,54,85$ and 87 SIAs. Bcc and non-bcc atoms identified by the CNA method are shown by grey dots and blue spheres respectively.

simulations are performed. In each simulation, a C15 cluster of 5 SIAs is initially created, and individual SIAs are randomly inserted one at a time, by inserting a $\langle 110\rangle$ dumbbell at a randomly-chosen atomic site 0.5 to $1.0 \mathrm{~nm}$ away from the initial cluster. Following each insertion, the system is relaxed for $250 \mathrm{ps}$ at $300 \mathrm{~K}$ for the newly inserted SIA to diffuse to the cluster.

In growth simulations all $\mathrm{C} 15$ clusters grow firstly by absorbing SIAs, as expected; however, they all transform into loops later, as shown in Fig. 2. Both $\langle 100\rangle$ and $\langle 111\rangle$ loops are possible products, indicating the stochastic nature. Two loops obtained from the transformation, one $\langle 100\rangle$ and one $\langle 111\rangle$, have been annealed at $300 \mathrm{~K}$ for another 2 ns without observing further structural change. Their stability is also indicated by the convergence of total potential energy. Among all 40 growth simulations, $70 \%$ (29 out of 40) transform into $\langle 100\rangle$ loops and $30 \%$ into $\langle 111\rangle$ loops. The critical sizes for the transformation to occur fluctuate from 56 to 147 SIAs (1.3-1.9 nm in diameter) in these simulations. Since the existence of C15 clusters was predicted only very recently [18], so far no experimental effort has been reported to characterize them. Moreover, driven by the high strain and excess cohesive energies, C15 clusters transform into loops at sizes below $2 \mathrm{~nm}$, making full experimental characterization difficult.

More importantly, the growth simulations reveal a new mechanism for $\langle 100\rangle$ loop formation - by transformation from C15 clusters. The detailed transformation mechanisms are further explored by 20 annealing simulations. In each simulation, a C15 cluster of 95 SIAs is coherently inserted into a bcc matrix, followed by 0.5 ns relaxation at $900 \mathrm{~K}$. Consistent with growth simulations, a C15 cluster may transform into either a $\langle 100\rangle$ or a $\langle 111\rangle$ loop during annealing. Sim- ilarly, either $\langle 100\rangle$ or $\langle 111\rangle$ loop may result from the interactions of two $\langle 111\rangle$ loops [11]. As examples, two transformation processes are demonstrated in Fig. 3, with the final products being a $[010]$ and a $[\overline{1} 11] / 2$ loop, respectively. Here we use the dislocation-extraction-algorithm [24] to identify the loop structures and Burgers vectors, with the results confirmed by the corresponding atomic configurations (insets in Fig. 3). The transformation begins with the nucleation of $\langle 111\rangle$ type segments which evolve into complex loop configurations containing possibly both $\langle 111\rangle$ and $\langle 100\rangle$ components. These segments further react with each other to reduce the total loop length and thus energy, until a single loop forms.

The nucleation of $\langle 111\rangle$ segments is stochastic in nature, with all four equivalent Burgers vectors possible. After nucleation, the subsequent interactions are found to follow conventional dislocation reaction. As shown Fig. 3(a), the transformation to a [010] loop starts with the nucleation of a $[\overline{1} 1 \overline{1}] / 2$ segment at $120 \mathrm{ps}$. At $125 \mathrm{ps}$, a loop complex forms with 6 segments, including two $\langle 100\rangle$ ones. The [010] segment forms by the reactions:

$$
\begin{gathered}
{[\overline{1} 1 \overline{1}] / 2+[111] / 2=[010]} \\
{[\overline{1} \overline{1} 1] / 2+[1 \overline{1} \overline{1}] / 2=-[010]}
\end{gathered}
$$

And the $[\overline{1} 00]$ segment by:

$$
[\overline{1} 1 \overline{1}] / 2+[\overline{1} \overline{1} 1] / 2=[\overline{1} 00]
$$

At $126 \mathrm{ps}$, the [100] segments vanishes via the reverse of reaction 5 , and the $[111] / 2$ and $[\overline{1} 1 \overline{1}] / 2$ segments react into the [010] segment by reaction 3 . The two $\langle 111\rangle$ segments remained at 126 ps continue to react via reaction 4 and eventually, a single [010] loop forms. A similar reaction path for $\langle 111\rangle$ loop formation is shown in Fig. 3 (b).

Besides the M07 potential, the growth simulations have been repeated using the Ackland 97 [25], Mendelev [26], Ackland 04 [27] and the magnetic potential (MP-cs3-30) [28] for possible effect from magnetic contribution, 20 simulations for each potential. In all simulations, initial growth of C15 clusters and subsequent transformation into either $\langle 111\rangle$ or $\langle 100\rangle$ loops are observed. In comparison, the M07 potential predicts higher probability for $\langle 100\rangle$ loop formation, with also relatively larger critical transition sizes. The other potentials underestimate the stability of $\mathrm{C} 15$ clusters in reference to DFT calculations [18], and their chances to grow 

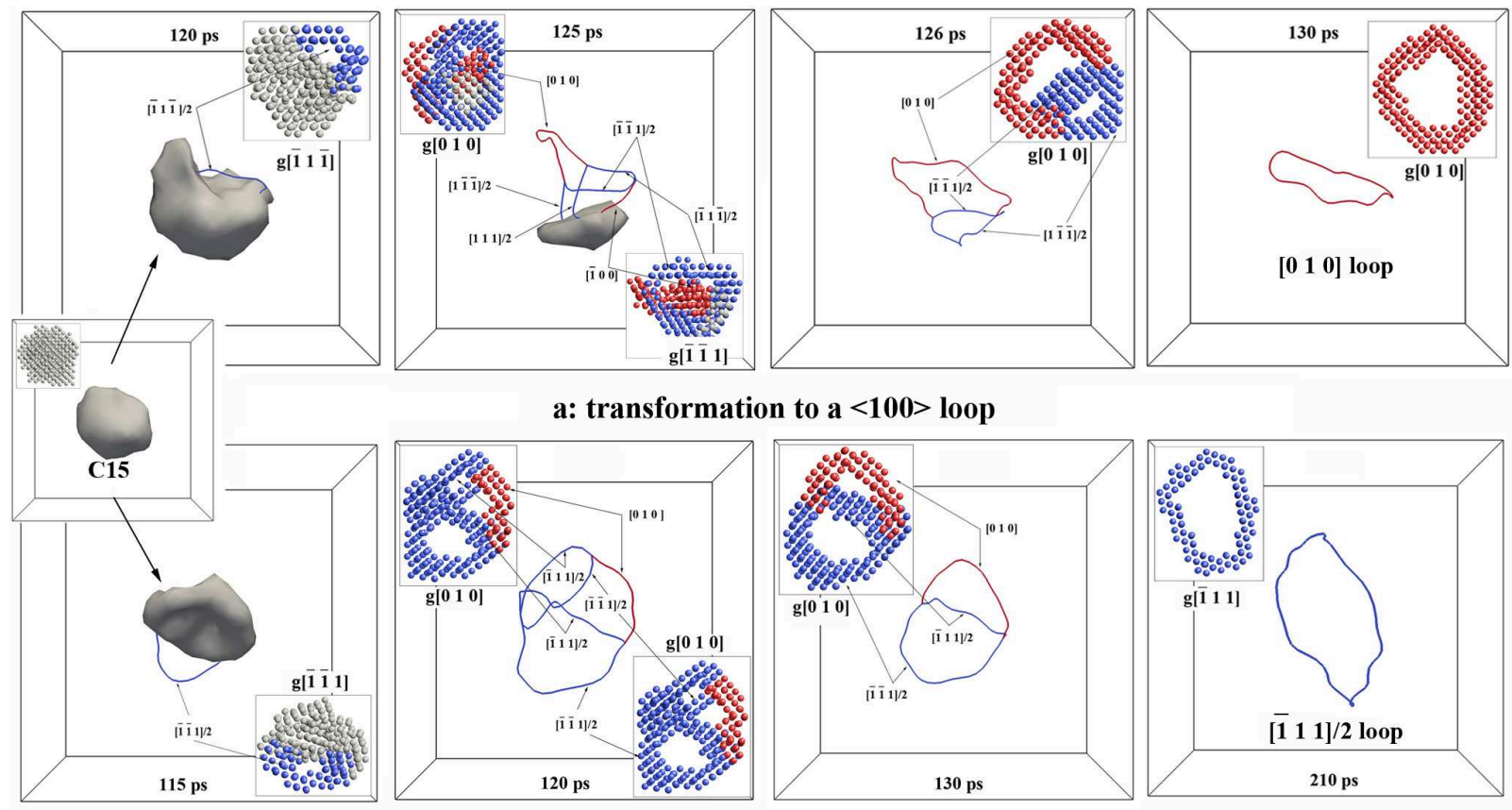

a: transformation to a $<\mathbf{1 0 0}>$ loop
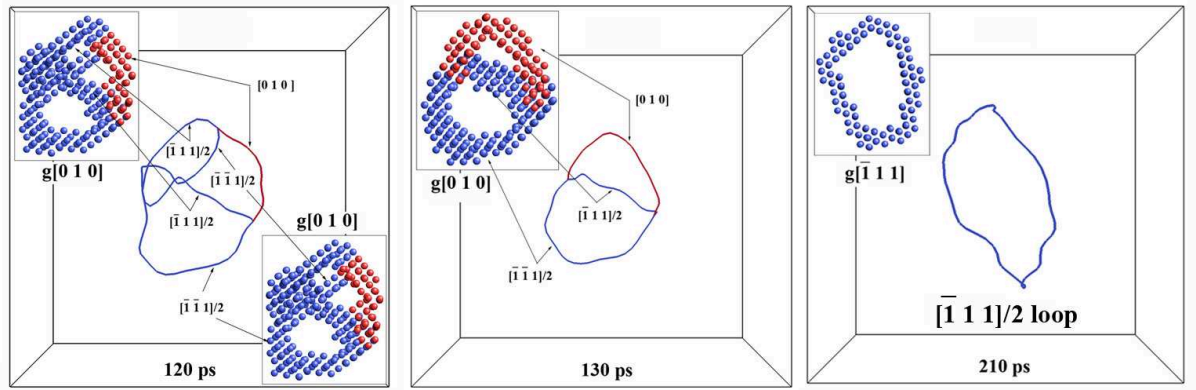

b: transformation to a $<111>$ loop

Figure 3: (Color online) Transformation paths from a C15 cluster (95 SIAs) to a (a) [010] loop and (b) [1111]/2 loop at 900 $\mathrm{K}$. Insets are the corresponding atomic configurations projected along vector g. Only non-bcc atoms are shown, with those associated with $\langle 100\rangle$ segments $(\langle 111\rangle)$ colored in red (blue), and others in gray.

into large sizes. Surveying literature, formation of 3D SIA clusters have been predicted by various potentials $[15,16,18,29]$, with possible transformation into glissile loops [30, 31]. Therefore, the above mechanisms are not specific to the M07 potential.

C15 clusters may form directly in displacement cascades [18], e.g., produced by ion or neutron irradiation. In a separate simulation we found that they can also form by clustering of individual SIAs, suggesting that they may also form under electron irradiation. They have important impact on longterm defect evolution in bcc steels for their superior stability at small sizes and subsequent transformation into loops. Furthermore, a new mechanism is suggested for $\langle 100\rangle$ loop formation - by clustering of SIAs, with $\mathrm{C} 15$ clusters being the intermediate stages. This process does not require pre-existing $\langle 111\rangle$ loops, which are necessary in all previous proposed mechanisms $[8,11,12]$. Consistently, recent experiments showed that under ion-irradiation $\langle 100\rangle$ loops form exclusively without presence of $\langle 111\rangle$ loops at temperatures above $500^{\circ} \mathrm{C}[4]$. Therefore, the new mechanism may provide a better explanation to the experiments, and may motivate direct experimental validation.
The authors thank the support of the DOE Light Water Reactor Sustainability Program.

\section{References}

[1] B. C. Masters, Philos. Mag. 11, 881 (1965).

[2] B. L. Eyre and R. Bullough, Philos. Mag. 12, 31 (1965).

[3] S. L. Dudarev, R. Bullough, and P. M. Derlet, Phys. Rev. Lett. 100, 135503 (2008).

[4] Z. Yao, M. L. Jenkins, M. Hernandez-Mayoral, and M. A. Kirk, Philos. Mag. 90, 4623 (2010).

[5] B. D. Wirth, G. R. Odette, D. Maroudas, and G. E. Lucas, J. Nucl. Mater. 276, 33 (2000).

[6] Y. N. Osetsky, D. J. Bacon, A. Serra, B. N. Singh, and S. I. Golubov, J. Nucl. Mater. 276, 65 (2000).

[7] K. Arakawa, K. Ono, M. Isshiki, K. Mimura, M. Uchikoshi, and H. Mori, Science 318, 956 (2007).

[8] J. Marian, B. D. Wirth, and J. M. Perlado, Phys. Rev. Lett. 88, 255507 (2002).

[9] C. C. Fu, F. Willaime, and P. Ordejon, Phys. Rev. Lett. 92, 175503 (2004).

[10] L. Malerba, M. C. Marinica, N. Anento, C. Bjrkas, H. Nguyen, C. Domain, F. Djurabekova, P. Olsson, K. Nordlund, A. Serra, D. Terentyev, F. Willaime, and C. S. Becquart, J. Nucl. Mater. 406, 19 (2010).

[11] H. X. Xu, R. E. Stoller, Y. N. Osetsky, and D. Terentyev, Phys. Rev. Lett. 110, 265503 (2013).

[12] K. Arakawa, M. Hatanaka, E. Kuramoto, K. Ono, and H. Mori, Phys. Rev. Lett. 96, 125506 (2006).

[13] K. Arakawa, T. Amino, and H. Mori, Acta Mater. 59, 141 (2011). 
[14] J. Chen, N. Gao, P. Jung, and T. Sauvage, J. Nucl. Mater. 441, 216 (2013).

[15] R. E. Stoller, J. Nucl. Mater. 233, 999 (1996).

[16] F. Gao, D. J. Bacon, Y. N. Osetsky, P. E. J. Flewitt, and T. A. Lewis, J. Nucl. Mater. 276, 213 (2000).

[17] D. A. Terentyev, T. P. C. Klaver, P. Olsson, M. C. Marinica, F. Willaime, C. Domain, and L. Malerba, Phys. Rev. Lett. 100, 145503 (2008).

[18] M. C. Marinica, F. Willaime, and J. P. Crocombette, Phys. Rev. Lett. 108, 025501 (2012).

[19] L. Dzerald, M. C. Marinica, L. Ventelon, D. Rodney, and F. Willaime, J. Nucl. Mater. 449, 219 (2014).

[20] S. Plimpton, J. Comp. Phys. 117, 1 (1995).

[21] J. D. Honeycutt and H. C. Andersen, J. Phys. Chem. 91, 4950 (1987).

[22] D. J. Bacon, R. Bullough, and J. R. Willis, Philos. Mag. 22, 31 (1970).

[23] J. D. Eshelby, Solid State Physics 3, 79 (1958).

[24] A. Stukowski, V. V. Bulatov, and A. Arsenlis, Modelling Simul. Mater. Sci. Eng. 20, 085007 (2012).

[25] G. J. Ackland, D. J. bacon, A. F. Calder, and T. Harry, Phil. Mag. A 75, 713 (1997).

[26] M. I. Mendelev, S. Han, D. J. Srolovitz, G. J. Ackland, D. Y. Sun, and M. Asta, Philos. Mag. 83, 3977 (2003).

[27] G. J. Ackland, M. I. Mendelev, D. J. Srolovitz, S. Han, and A. Barashev, J. Phys. Condens. Matter 16, S2629 (2004).

[28] S. Chiesa, P. M. Derlet, and S. L. Dudarev, Phys. Rev. B 79, 214109 (2009).

[29] D. Terentyev, L. Malerba, P. Klaver, and P. Olsson, J. Nucl. Mater. 382, 126 (2008).

[30] H. X. Xu, R. E. Stoller, and Y. N. Osetsky, J. Nucl. Mater. 443, 66 (2013).

[31] Y. Fan, A. Kushima, and B. Yildiz, Phys. Rev. B 81, $104102(2010)$ 